\title{
Diseño biomecánico del puesto de trabajo de noqueo en el faenamiento de ganado
}

\section{Biomechanical design of the knockout workstation in cattle slaughter}

Manolo Alexander Córdova Suárez. ${ }^{1}$, Luis Javier Tapia Vasco. ${ }^{2}$, Zoila Eliana Zambrano Ochoa. ${ }^{3}$ \& Juan Pablo Muquinche Puca. ${ }^{4}$

Recibido: 01-06-2020 / Revisado: 15-06-2020 / Aceptado: 03-07-2020 / Publicado: 04-08-2020

\begin{abstract}
.
DOI: https://doi.org/10.33262/concienciadigital.v3i3.2.1398

Productive activities with exposure to static load cause many musculoskeletal disorders, this work analyzes and ergonomically structures the cattle slaughter workstation in the humid area of Camal in the Municipality of Ambato, for this it establishes a significance analysis of the variables with BIO-mec computer tool. This research begins with a work study through field observation to identify the activities, tasks, execution times related to the cattle knockout position in the Ambato Municipal Camal in the cattle slaughter area that are related to the management manual loading and the adoption of an uncomfortable posture in its execution that can cause overload of corporal dispositions such as: joints, tendons, tendon sheaths, ligaments, muscles in the worker. Then a significance analysis of the variables determined by the BIO-mec method is carried out considering characteristics: physiological of the worker, weight of the sustained load, critical posture of the worker, duration of the efforts, angles of the body segments and the percentage of the maximum bearable load reached in each joint to establish their importance and modify the job position until complying with the limits established by Ecuadorian legislation. The design result determines a negative impact on the maximum permissible loads of elbow, shoulder, torso, hip, knee and ankle with the use of a platform adjustable to the size of the cattle, improving the posture angles of:
\end{abstract}

${ }^{1}$ Regional Autonomous University of Los Andes, Postgraduate, Ambato, Ecuador, pg.docentemcs@uniandes.edu.ec

${ }^{2}$ Department of Food Science and Engineering, Technical University of Ambato, Ambato, Ecuador,

lj.tapia@uta.edu.ec

${ }^{3}$ Faculty of Agroindustrial Engineering, Technical University of Cotopaxi, Latacunga, Ecuador, zoila.zambrano@utc.edu.ec

${ }^{4}$ Abreka Ingeniería Cía. Ltda., Ambato, Ecuador, jp.muquinche@gmail.com 


\section{ISSN: 2600-5859}

$68.6^{\circ}$ for the ankle, $132^{\circ}$ for knee, $92^{\circ}$ hip, $45^{\circ}$ for L5 / S1, $51^{\circ}$ for shoulder, $36^{\circ}$ for wrist. With a suspended load of $4 \mathrm{~kg}$. For a worker of $170 \mathrm{~cm}, 75 \mathrm{~kg}$ of weight and of masculine gender.

Keywords: Ergonomic risks, biomechanics, Maximum admissible load.

\section{Resumen.}

Las actividades productivas con exposición a carga estática causan muchos trastornos músculo esquelético, este trabajo analiza y estructura ergonómicamente el puesto de trabajo de faenamiento de ganado en la zona húmeda del Camal del Municipio de Ambato, para ello establece un análisis de significación de las variables con herramienta informática BIO-mec. Esta investigación empieza con un estudio de trabajo mediante observación de campo para identificar las actividades, tareas, tiempos de ejecución relacionados con el puesto de noqueo de reses en el Camal Municipal de Ambato en la zona de faenamiento de ganado vacuno que se relacionan con el manejo manual de carga y la adopción de postura incomoda en su ejecución que pueden causar sobrecarga de disposiciones corporales como: articulaciones, tendones, vainas tendinosas, ligamentos, músculos en el trabajador. Luego se realiza un análisis de significación de las variables que determina el método BIO-mec considerando características: fisiológicas del trabajador, peso de la carga sostenida, postura crítica del trabajador, duración de los esfuerzos, ángulos de los segmentos corporales y el porcentaje de la carga máxima soportable alcanzado en cada articulación para establecer la importancia de las mismas y modificar el puesto de trabajo hasta cumplir con los límites establecidos por la legislación Ecuatoriana. El resultado del diseño determina una afectación negativa en las cargas máximas admisibles de codo, hombro, torso, cadera, rodilla y tobillo con el uso de una plataforma ajustable al tamaño del ganado, mejorando los ángulos de postura de: $68,6^{\circ}$ para el tobillo, $132^{\circ}$ para rodilla, $92^{\circ}$ cadera, $45^{\circ}$ para $\mathrm{L} 5 / \mathrm{S} 1,51^{\circ}$ para hombro, $36^{\circ}$ para muñeca. Con una carga suspendida de $4 \mathrm{~kg}$. Para un trabajador de $170 \mathrm{~cm}, 75 \mathrm{~kg}$ de peso y de género masculino.

Palabras claves: Riesgos ergonómicos, biomecánica, Carga máxima admisible.

\section{Introducción.}

De todos los trabajadores de camales de faenamiento municipales ecuatoriano que ejecutan las actividades de noqueo el $90 \%$ ha sufrido trastornos musculo esqueléticos.

El uso de medidas ergonómicas ha demostrado ser útil para reducir la exposición a factores de riesgo como: manejo de carga estáticas y posturas de trabajo incómodas que pueden causar no solo enfermedades sino accidentes por la disconfort que existe en estos puestos de trabajo(Jensen \& Friche, 2008). Para atenuar el esfuerzo de la postura y las sobrecargas en las articulaciones, codos, manos, antebrazos, espalda y piernas causadas por los esfuerzos causados por los malos diseños 
de los puestos de trabajo o simplemente por la adaptación de la persona al puesto de trabajo se debe aplicar controles con fundamentos de biomecánica.(Dufour \& Pillu, 2018)

Para reducir la exposición a factores de riesgo ergonómicos se utilizan diferentes estrategias, como informativas, capacitación y entrenamiento y estrategias de facilitación(Ardila Jaimes \& Rodríguez, 2013). Estas estrategias o combinaciones de estrategias intervienen en el proceso de reducción factores de riesgo ergonómicos, pero a veces no son suficientes si no se considera la modificación del puesto debido a las restricciones propias del trabajo que involucran modificar procesos o más personal. Es por ello que una alternativa es el análisis ergonómico para hacer una reingeniería del puesto de trabajo y determinar la mejor alternativa para evitar los efectos nocivos de estos puestos de alto riesgo (Casanova Párraga \& De la Cruz Moreta, 2017)

Los trabajadores de faenamiento vacuno en el área de noqueo utilizan herramientas que llegan a pesar hasta $15 \mathrm{~kg}$ que ocupan plataformas elevadas como piso que no se adaptan a las medidas antropométricas del trabajador (Figueroa \& Abel, 2010) causando molestias tempranas relacionadas a trastornos musculo-esqueléticos y hasta accidentes significativos por la exposición a movimientos bruscos y reses muy grandes que suben a su plano de trabajo y causan accidentes,

En la actualidad, la exigencia profesional en cuanto a diseño de puestos de trabajo recomienda el diseño al inicio de la actividad productiva, pero el crecimiento de la capacidad (Macedo, Issa, Defino, \& Shimano, 2015)y las nuevas exigencias propias de cada proceso productivo obligan realizar modificaciones que si no son justificadas incurren en gastos innecesarios(Gil Agudo, 2019). El análisis de significación establece un orden de importancia de las variables a modificar que da una garantía de evitar errores en el diseño de cualquier prototipo o en este caso un puesto de trabajo.(Robaina Castellanos, Medina, Manuel, Morales Rigau, \& Robaina Castellanos, 2001)

Este trabajo refleja la aplicación de la biomecánica en la mejora de puestos artesanales que nadie considera pese a la importancia en convivir diario por el consumo de carne.

\section{Metodología.}

\section{Estudio de trabajo.}

Para determinar la eficiencia de las actividades en la ejecución de un proceso productivo se debe realizar una observación directa de toda la cadena productiva y poder identificar las actividades, tareas, tiempos de ejecución de los procesos, subprocesos, ciclos, relacionados con la manipulación de cargas estáticas relacionadas al faenamiento de ganado vacuno además se debe determinar aspectos fisiológicos del trabajador como: género, edad, el peso del trabajador y la carga, estatura del trabajado, soporte de la carga o fuerza, la frecuencia que adopta el trabajador en la postura que se analizará y las dimensiones antropométricas que se generarán tras la postura del trabajador. Para este trabajo se determinaron las condiciones de proceso analizado, Los pesos 
por extremidades del trabajador, y datos adicionales como coeficiente de fricción y porcentaje de la población a proteger.

\section{Evaluación del levantamiento de cargas por el método de BIO-mec.}

Con el fin de mejorar la calidad de vida de los trabajadores, los estudios multidisciplinarios cada vez son más frecuentes en un intento por mejorar la biomecánica. Uno de los métodos de análisis con mayor precisión es el que recomienda la Universidad de valencia en su herramienta BIO-mec de ergo-nautas (Mira, Cardona, Vargas, \& Buitrago, 2020) que estudia el comportamiento de manera física calculando la carga estática postural recomendada y crítica para cada parte del cuerpo sujeta a tensión y torsión por la ejecución a las tareas de manera estática (Remesal, 2004).

Luego de procesar la información propia de cada puesto en cuanto a ángulos y peso que sujeta la persona de manera estática la herramienta calcula el peso de cada sección y longitud del trabajador Además determina si existe o no afectación en alguna articulación (Castillo, 2014).

\section{Análisis de significación de variables.}

En esta etapa de la investigación se realizó un análisis de significación de las variables involucradas manteniendo los datos antropométricos del trabajador constante, así como las dimensiones del plano de trabajo donde se asienta el noqueador de la res que actúa en el área de faenamiento de ganado. Se empezó analizando los elementos sujetos a modificación en el puesto de trabajo: peso de la carga, altura y distancia del levantamiento; y se mantuvo las partes del puesto de trabajo que por sus características no se pueden modificar como: el tiempo y la frecuencia en que se realizan los esfuerzos, género del trabajador.

Al final se realiza iteraciones sucesivas para determinar la variable con mayor influencia en el resultado final de la afectación a la o las articulaciones afectadas con la ayuda de la herramienta BIO-mec.(Ardila Jaimes \& Rodríguez, 2013). Se debe llenar la tabla 1. Con los resultados para cada caso.

Tabla 1. Cargas máximas recomendables, sobrecarga y riesgo por articulación

\begin{tabular}{|c|c|c|c|c|}
\hline Ítem & Peso de la carga & $\begin{array}{c}\text { Articulación } \\
\text { analizada }\end{array}$ & $\begin{array}{c}\text { Sobre } \\
\text { Carga }(k g)\end{array}$ & Afectación \\
\hline $\begin{array}{l}\text { Primera } \\
\text { iteración }\end{array}$ & & $\begin{array}{c}\text { Codo } \\
\text { Hombro } \\
\text { Lumbar } \\
\text { Cadera } \\
\text { Rodilla } \\
\text { Tobillo }\end{array}$ & & SI/NO \\
\hline
\end{tabular}

Fuente: Elaboración propia. 


\section{Resultados y discusión.}

\section{Resultados del estudio de trabajo.}

En la Tabla $\mathrm{N}^{\circ} 1$ se observa el resultado del estudio de trabajo realizado con observación de campo durante periodos de trabajo en condiciones de carga máxima de producción.

Tabla 1. Datos obtenidos para el estudio del puesto de trabajo

\begin{tabular}{|c|c|c|}
\hline Dimensión & ITEM & Valor \\
\hline \multirow{4}{*}{ Datos del puesto } & Departamento/ Área & Faenamiento \\
\hline & Puesto analizado & Noqueo \\
\hline & Duración de la postura & Menos de 1 hora \\
\hline & Repetición de ciclos & $\begin{array}{c}\text { Menos de } 1 \text { vez cada } 5 \\
\text { minutos }\end{array}$ \\
\hline \multirow{14}{*}{$\begin{array}{c}\text { Pesos de las extremidades } \\
\text { del trabajador }{ }^{\text {a }}\end{array}$} & Ejecución de la tarea & Con una sola mano \\
\hline & Peso del trabajador & $70 \mathrm{~kg}$ \\
\hline & Peso de la cabeza & $4,65 \mathrm{~kg}$ \\
\hline & Peso del cuello & $1,65 \mathrm{~kg}$ \\
\hline & Peso tórax & $16,43 \mathrm{~kg}$ \\
\hline & Peso abdomen & $11,03 \mathrm{~kg}$ \\
\hline & Pesos pelvis & $10,05 \mathrm{~kg}$ \\
\hline & Peso brazo & $2,10 \mathrm{~kg}$ \\
\hline & Peso antebrazo & $1,27 \mathrm{~kg}$ \\
\hline & Peso mano & $0,45 \mathrm{~kg}$ \\
\hline & Peso muslo & $7,5 \mathrm{~kg}$ \\
\hline & Peso pierna & $3,23 \mathrm{~kg}$ \\
\hline & Peso pie & $1,05 \mathrm{~kg}$ \\
\hline & Coeficiente de rozamiento & $0,5^{\mathrm{b}}$ \\
\hline Condiciones adicionales & $\begin{array}{l}\text { Porcentaje de la población a } \\
\text { proteger }\end{array}$ & $90 \%{ }^{c}$ \\
\hline
\end{tabular}

Nota: ${ }^{\mathrm{a}}$ Los pesos son estimados a partir del peso del individuo. ${ }^{\mathrm{b}}$ Coeficiente de rozamiento entre la suela del calzado y el suelo (Yan, 2017) ${ }^{\mathrm{c}}$ Porcentaje de población que resulta protegida en los resultados obtenidos. La estatura del trabajador es de 1,65 $\mathrm{m}$ y la postura dura menos de una hora.

Fuente: Elaboración propia.

La postura crítica del puesto de noqueador de reses identificada luego del estudio de trabajo es inclinada de frente a la cabeza del ganado, la flexión de la mano derecha con la pistola neumática la que soporta el peso de la carga sostenida como se muestran en las Fig. 1 
Figura 1. Posición crítica en puesto de noqueo

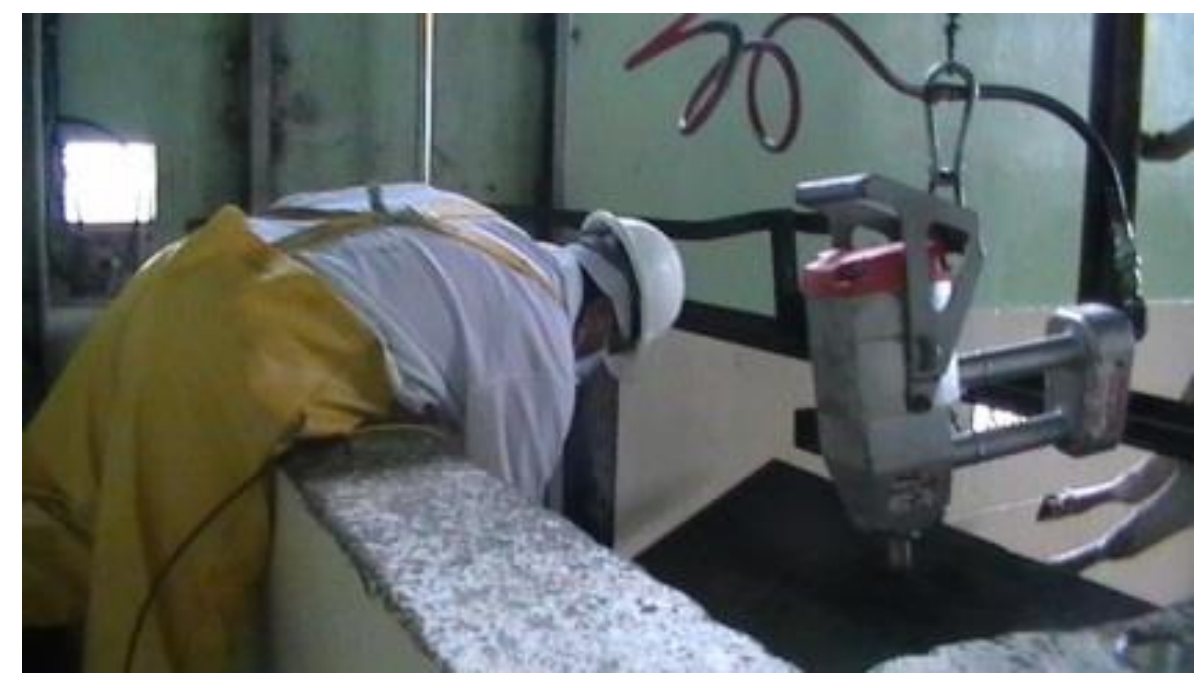

Fuente: Elaboración propia.

\section{Resultados de la evaluación del puesto de trabajo con el método BIO-mec.}

En el gráfico $\mathrm{N}^{\circ} 1$ se observan los resultados del porcentaje de la carga máxima soportable alcanzado en cada articulación del trabajador de Noqueo de ganado.

Figura 2. Porcentaje de la carga máxima admisible para cada extremidad del noqueador de ganado. Adaptado BIO-mec.

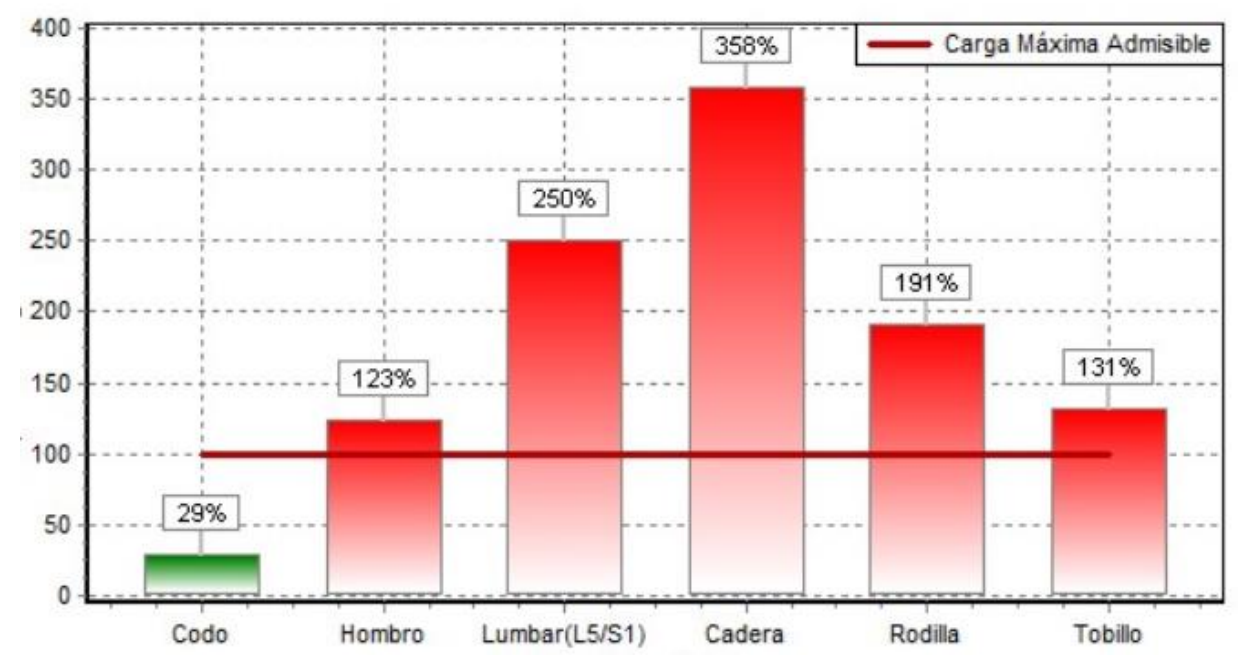

Fuente: Elaboración propia.

En la tabla 2, se presenta los resultados de las cargas máximas, la sobrecarga y el riesgo para el trabajador según cada articulación en la postura crítica analizada y sin mejoras. La limitación viene 
impuesta por la articulación de la Cadera, que es la menos carga puede soportar en estas condiciones y se determina que si existe riesgo por sobre esfuerzo.

Tabla 2. Cargas máximas recomendables, sobrecarga y riesgo por articulación.

\begin{tabular}{|c|c|c|c|c|}
\hline $\begin{array}{c}\text { Articulación } \\
\text { estudiada }\end{array}$ & $\begin{array}{c}\text { CMR } \\
(\mathrm{kg})\end{array}$ & $\begin{array}{c}\text { SC } \\
(\mathrm{kg})\end{array}$ & Riesgo* & $\begin{array}{c}\text { \% Pob-Prot } \\
(\%)\end{array}$ \\
\hline Codo & 52. & $-37,6$ & No & 100 \\
\hline Hombro & 12,21 & 2,79 & Sí & 95,3 \\
\hline $\begin{array}{l}\text { Lumbar } \\
\text { (L5/S1) }\end{array}$ & 5,99 & 9,01 & Sí & 79,6 \\
\hline Cadera & 4,19 & 10,81 & Sí & 80,5 \\
\hline Rodilla & 7,84 & 7,16 & Sí & 85,4 \\
\hline Tobillo & 11,48 & 3,52 & Sí & 90,6 \\
\hline
\end{tabular}

Fuente: Elaboración propia.

Nota: $\mathrm{CMR}=$ Carga máxima recomendable. $\mathrm{SC}=$ Sobrecarga. \%Prob-pot: Porcentaje de trabajadores que no encontrarían problemas en esta situación si el esfuerzo fuera puntual. Riesgo* $^{*}$ existencia de riesgo por sobreesfuerzos. Se considera que existe riesgo cuando la sobrecarga sea positiva.

La postura resultante se observa en la figura 3.

Figura 3. Postura resultante al procesar en el programa Kinovea

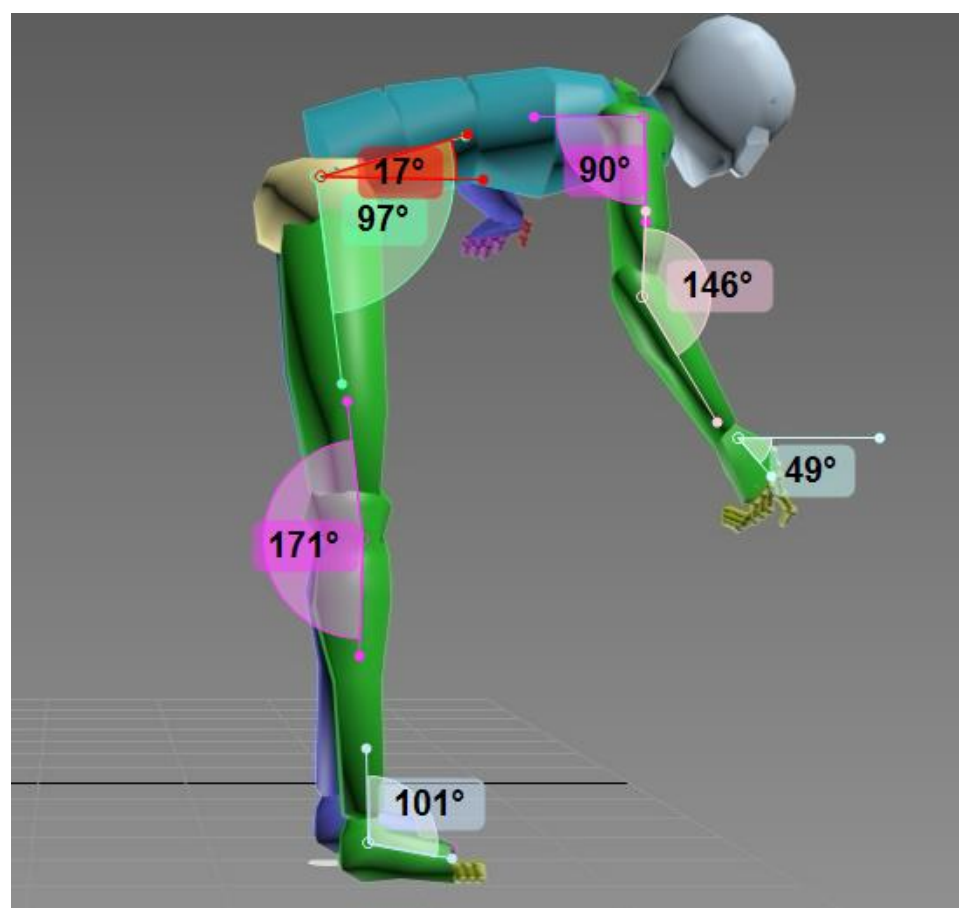

Fuente: Elaboración propia. 
ISSN: 2600-5859

\section{Resultado del análisis de significación.}

Luego de establecer las cargas máximas se evaluó el porcentaje de la carga máxima admisible en el puesto de trabajo de noqueo del área de faenamiento de ganado. En la tabla 3 se muestra el análisis de significación de las variables y su incidencia en la sobre carga detectada estimados por el software BIO-MEC hasta eliminar la afectación de la articulación de la cadera.

Tabla 3. Cargas máximas recomendables, sobrecarga y riesgo por articulación.

\begin{tabular}{|c|c|c|c|c|}
\hline Personal & PC & Articulación & $\begin{array}{c}\text { Sobre } \\
\text { Carga (kg) }\end{array}$ & Afectación \\
\hline \multirow{7}{*}{$\begin{array}{l}\text { Primera } \\
\text { iteración }\end{array}$} & \multirow{7}{*}{10} & Codo & $-42,6$ & $\mathrm{NO}$ \\
\hline & & Hombro & $-2,21$ & NO \\
\hline & & Lumbar & 4,01 & SI \\
\hline & & Cadera & 5,81 & SI \\
\hline & & Rodilla & 2,16 & SI \\
\hline & & Tobillo & $-1,48$ & $\mathrm{NO}$ \\
\hline & & Codo & $-47,6$ & NO \\
\hline \multirow{4}{*}{$\begin{array}{l}\text { Segunda } \\
\text { iteración }\end{array}$} & \multirow{4}{*}{5} & Hombro & $-7,21$ & $\mathrm{NO}$ \\
\hline & & Lumbar & $-0,99$ & NO \\
\hline & & Cadera & 0,81 & SI \\
\hline & & Rodilla & $-2,84$ & NO \\
\hline \multirow{7}{*}{$\begin{array}{l}\text { Tercera } \\
\text { iteración }\end{array}$} & \multirow{7}{*}{4,5} & Tobillo & $-6,48$ & $\mathrm{NO}$ \\
\hline & & Codo & $-48,1$ & NO \\
\hline & & Hombro & $-7,71$ & NO \\
\hline & & Lumbar & $-1,49$ & $\mathrm{NO}$ \\
\hline & & Cadera & 0,31 & SI \\
\hline & & Rodilla & $-3,34$ & $\mathrm{NO}$ \\
\hline & & Tobillo & $-6,98$ & NO \\
\hline \multirow{6}{*}{$\begin{array}{l}\text { Cuarta } \\
\text { iteración }\end{array}$} & \multirow{6}{*}{4} & Codo & $-48,6$ & NO \\
\hline & & Hombro & $-8,21$ & NO \\
\hline & & Lumbar & $-1,99$ & $\mathrm{NO}$ \\
\hline & & Cadera & $-0,19$ & NO \\
\hline & & Rodilla & $-3,84$ & NO \\
\hline & & Tobillo & $-7,48$ & $\mathrm{NO}$ \\
\hline
\end{tabular}

Nota: $\mathrm{PC}=$ Carga máxima recomendable, Se fijó la distancia de traslado, género y antigüedad del trabajador en el puesto como variables constates. El peso de la carga se reduce por el uso de un sistema de amortiguamiento y una pistola neumática moderna. Los valores negativos determinan que no existe afectación.

Fuente: Elaboración propia.

Luego de establecer la carga máxima de la pistola neumática se recomienda el uso de una plataforma móvil en la base inferior del piso del ganado para ajustar los tamaños más bajos del ganado a las siguientes dimensiones determinadas por el programa BIO-mec en la tabla 4. 
Tabla 4. Dimensiones recomendadas

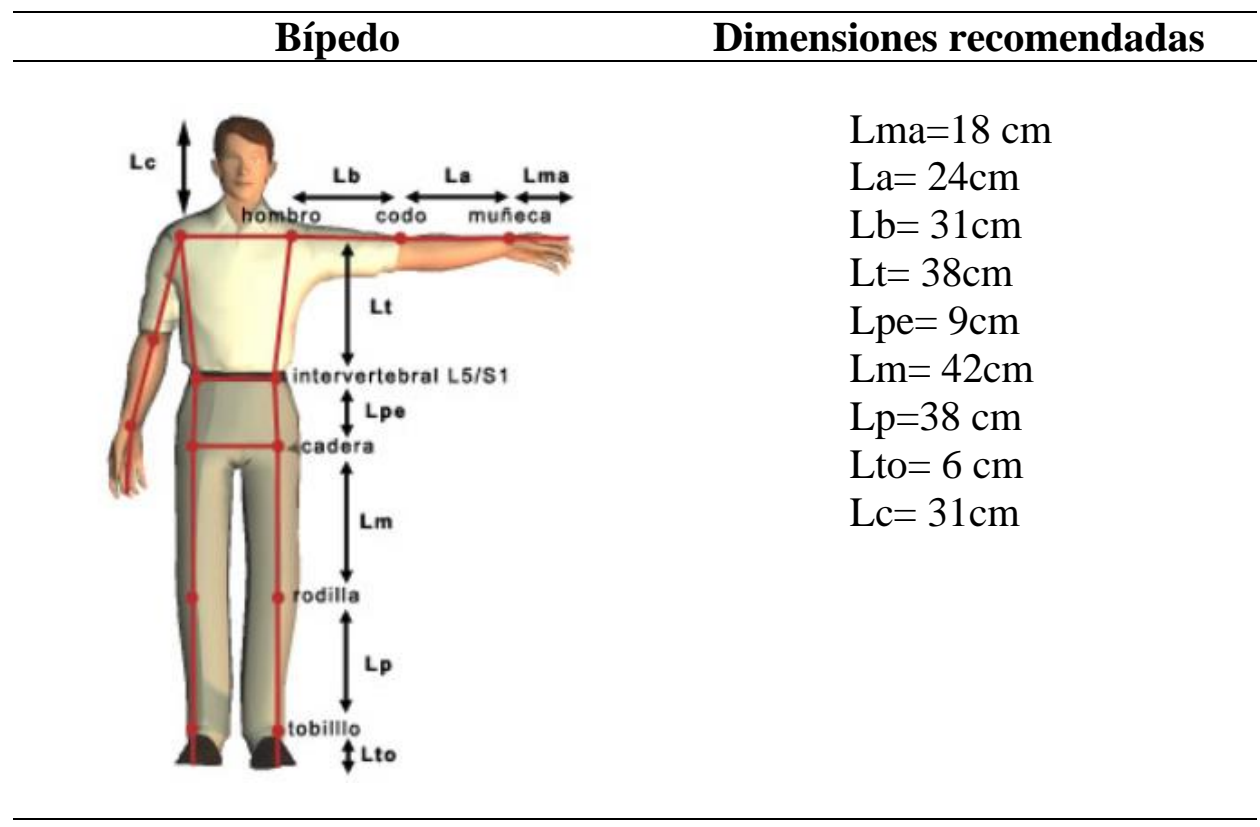

Fuente: Elaboración propia.

La postura recomendada se observa en la figura 4.

Figura 4. Postura recomendada al implementar pistola con yoyo de seguridad y plataforma

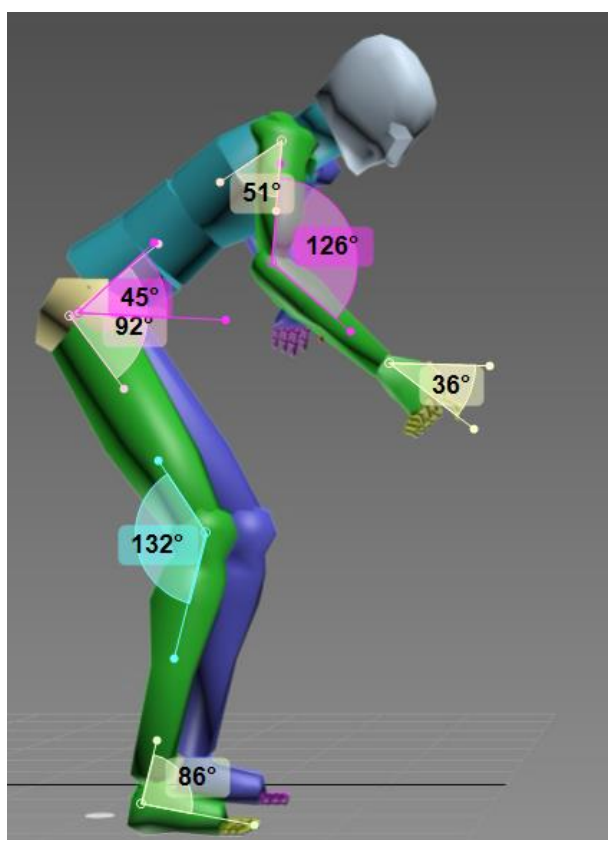

Fuente: Elaboración propia. 
Luego de establecer la carga máxima de la pistola neumática y el uso de la plataforma móvil los resultados de la sobre carga se observa en la figura 5.

Figura 5. Porcentaje de la carga máxima admisible para nuevo diseño de puesto de noqueo. Adaptado BIO-mec

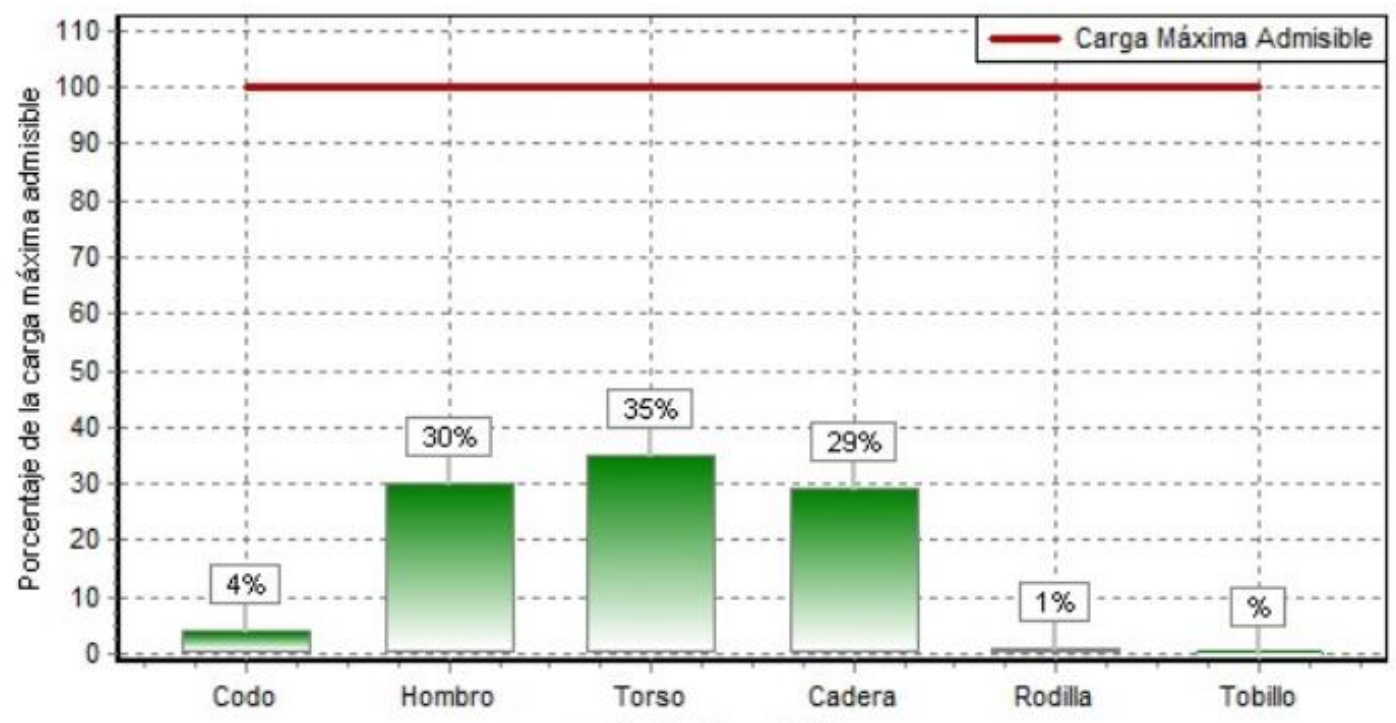

Fuente: Elaboración propia.

\section{Conclusiones.}

- Se diseñó un puesto de trabajo reduciendo el \% de carga máxima admisible de las articulaciones ha: $4 \%$ de codo, $30 \%$ de hombro, $35 \%$ de torso, $29 \%$ de cadera, $1 \%$ de rodilla y tobillo. Trabajando con una carga asistida por un sistema de suspensión aéreo de $4 \mathrm{~kg}$ en la pistola de noqueo y considerando una plataforma de elevación del ganado a faenar que ajuste los ángulos a: $68,6^{\circ}$ para el tobillo, $132^{\circ}$ para rodilla, $92^{\circ}$ cadera, $45^{\circ}$ para $\mathrm{L} 5 / \mathrm{S} 1$, $51^{\circ}$ para hombro, $36^{\circ}$ para muñeca; para una estación de trabajo de noqueo en la Sierra Ecuatoriana. Se redujo la afectación inicial por un mal diseño biomecánico en un $100 \%$.

\section{Referencias bibliográficas.}

Ardila Jaimes, C. P., \& Rodríguez, R. M. (2013). Riesgo ergonómico en empresas artesanales del sector de la manufactura, Santander. Colombia. Medicina y Seguridad del Trabajo, 59(230), 102-111.

Casanova Párraga, R. F., \& De la Cruz Moreta, E. F. (2017). Propuesta de mecanismos de prevención de riesgos laborales para la empresa pública municipal del Camal de Quevedo. Quevedo: UTEQ. 
Castillo, E. (2014). Evaluación de factores de riesgo ergonómicos e higiénicos en una empresa de recauchutado de neumáticos. Universidad de Alicante.

Dufour, M., \& Pillu, M. (2018). Biomecánica funcional. Miembros, cabeza, tronco: Elsevier. Figueroa, P., \& Abel, J. (2010). Proceso de faenamiento y menejo del ganado bovino y la incidencia en la contaminacion de las canales en el matadero Municipal del Cantón PAJAN. JIPIJAPA: UNESUM.

Gil Agudo, A. M. (2019). Biomechanical gait alterations: assessment protocol. Medicine (Spain), 12(75), 4462-4466. doi: 10.1016/j.med.2019.03.028

Jensen, L. K., \& Friche, C. (2008). Effects of training to implement new working methods to reduce knee strain in floor layers. A two-year follow-up. Occupational and Environmental Medicine, 65(1), 20-27.

Macedo, A. P., Issa, J. P. M., Defino, H. L. A., \& Shimano, A. C. (2015). Biomechanical evaluation of a spinal screw fixation system by the finite element method. International Journal of Morphology, 33(1), 318-326. doi: 10.4067/S0717-95022015000100050

Mira, N. O., Cardona, I. C. S., Vargas, K. C. C., \& Buitrago, P. A. R. (2020). Biomechanics of the dorsolumbar region during manual patient handling. Ciencia e Innovación en Salud.

Remesal, A. F. (2004). Análisis ergonómico de puestos de trabajo en el sector de calzado. Revista de biomecánica(42), 27-30.

Robaina Castellanos, G. R., Medina, P., Manuel, J., Morales Rigau, J. M., \& Robaina Castellanos, R. E. (2001). Análisis multivariado de factores de riesgo de prematuridad en Matanzas. Revista Cubana de obstetricia y ginecología, 27(1), 62-69.

Yan, X. (2017). Effects of friction property on biomechanics of lower limbs of table tennis players. Acta Technica CSAV (Ceskoslovensk Akademie Ved), 62(3), 29-36.

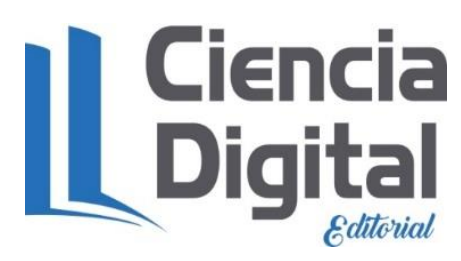


PARA CITAR EL ARTÍCULO INDEXADO.

Córdova Suárez, M. A., Tapia Vasco, L. J., Zambrano Ochoa, Z. E., \& Muquinche Puca, J. P. (2020). Diseño biomecánico del puesto de trabajo de noqueo en el faenamiento de ganado. ConcienciaDigital, 3(3.2), 6-17. https://doi.org/10.33262/concienciadigital.v3i3.2.1398

\section{【 Ciencia}

El artículo que se publica es de exclusiva responsabilidad de los autores y no necesariamente reflejan el pensamiento de la Revista Conciencia Digital.

El artículo queda en propiedad de la revista y, por tanto, su publicación parcial y/o total en otro medio tiene que ser autorizado por el director de la Revista Conciencia Digital.
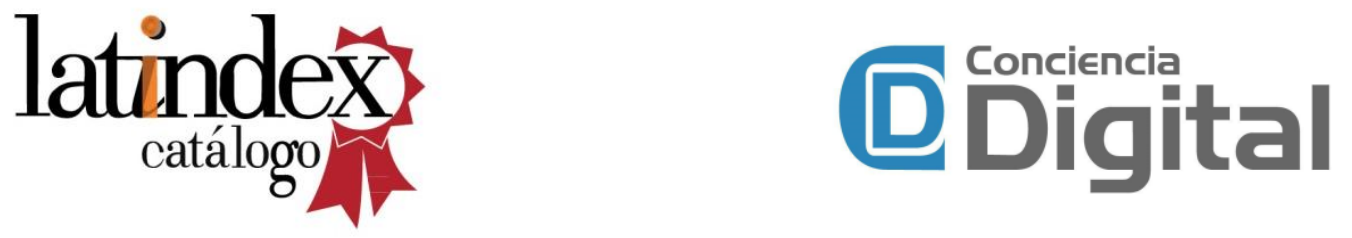